\title{
Raising Coverage and Capacity using Fixed Relays in a Realistic Scenario
}

\author{
Rainer Schoenen, Wolfgang Zirwas and Bernhard H. Walke
}

\begin{abstract}
Multihop techniques are known as a practical solution for covering huge radio cell areas when there are only very few base stations (BS). This is the case when fiber access is limited and BS CAPEX and OPEX are very expensive. For WiMAX and 3GPP-LTE it is possible to operate relay stations which are only fed over the air link. While having some inherent overhead due to increased radio resource usage, there are nevertheless impressive gains in the coverage compared to a $\mathrm{BS}$ alone and also in the capacity of the whole radio cell. Near relay nodes (RN) there is not only better SINR to the user, which is obvious, but it is often more efficient to associate to a RN instead of the BS, taking into account all resources used for the first and second hop. Therefore this is a low cost measure to increase the system efficiency. This has been shown in the literature for artificial scenarios so far.

In this paper we study a realistic scenario using data from topology information and raytracing. The city of Jersey was taken as example. The data is analyzed numerically, with all layer-1 and layer-2 performance models specified analytically. We study the case of one base station only, one BS with four RNs, and the latter plus another ring of nine RNs. The BS has fiber access, while the first hop of Relays (H1) is fed over the air from BS and the second hop $\mathrm{H} 2$ is fed by the RNs of group H1. We present results for the gains in coverage and capacity that are obtained by these Multihop techniques.
\end{abstract}

Index Terms-Relaying, Multihop, LTE, Coverage, Capacity

\section{INTRODUCTION}

$\mathbf{R}$ EALISTIC scenarios have not been analyzed so far for the use of fixed relay nodes in future OFDMA-based transmission schemes like 3GPP-LTE [1] or WiMAX [2]. The benefit for the coverage and capacity in relay enhanced radio cells was shown in the past with free-space propagation or Manhattan Grid [3]. Other related work in this area mostly analyzes regular cellular geometries without considering realistic pathloss due to obstructions [4]-[6]. In this paper we build upon 3D models of the city of Jersey [7] with raytracing tools [8], study the scenario for 2D and scalar performance measures and extend the analysis for realistic areas around the BS. Compared to abstract and regular cellular scenarios [9], this provides a good proof-of-concept.

The benefit of Multihop is the cost-efficient provisioning of radio access over the area, no matter if the BS is in lineof-sight or not. Using RNs is viable at much lower cost than providing more base stations due to their need for high-rate fixed network access at every location. Due to the limited power of transmitters and the high path loss in non-line-ofsight conditions the received signal strength is not sufficient

- Wolfgang Zirwas is with Siemens, Munich, Germany

- The author is with the Chair of Communication Networks at RWTH Aachen University, Faculty 6, Germany

- This work is funded by the BMBF ScaleNet project in Germany in many urban areas. To improve the coverage therefore more sending stations are needed, which can be full $B S$ s or relay nodes ( $R N \mathrm{~s}$ of store-and-forward type).

OFDMA-based transmission allows to coordinate radio resources in time and frequency domain. In any relay enhanced cell we assume a full coordination, so that there is no intra-cell interference. Therefore, a resource block can only be used by one of the actors, either $B S$ or one of the four $R N_{1} \in H 1$ or one of the nine $R N_{2} \in H 2$. For this reason, any traffic $P D U$ that goes from $B S$ to a user terminal $(U T)$ associated to $R N_{2}$ consumes three resource blocks $R_{0}, R_{1}, R_{2}$, one on each hop $0,1,2$. The size of $R_{i}$ in bits shall be the same, but the size in terms of time and frequency bandwidth $(T \times F)$ depends on the modulation\&coding scheme (PhyMode) on this subchannel.

Close to the sender, the higher received $S I N R$ value allows the highest PhyMode, i.e. the highest data rate. At the cell border the offered data rate is one order of magnitude lower (QPSK - 1/3 compared to QAM64 - 5/6 for LTE [1]). A terminal operating at the lowest PhyMode occupies a ten times higher part of the spectrum than a terminal operating at the highest PhyMode. That means the average cell capacity is overproportionally determined by the maximum possible rate at the outer regions.

The paper is structured as follows. Section II defines the scenario. Next, the used layer 1 (PHY) and layer 2 (DLC) models are explained. Numerical results presented in the next section, which also provides $2 \mathrm{D}$ result maps of the area. The last section ends with a concluding summary.

\section{Scenario Definition}

Jersey has been chosen for this scenario because the area of approx. $4.439 \mathrm{~km}^{2}$ is a typical cell size. By using a radio network planning tool, base stations and all relay nodes are placed at best possible locations, see Fig. 1. The topology (building placement) was known in advance and raytracing tools have been used to obtain the received signal power $P_{R, i}$ at each location from each possible transmitter site. The determination of the BS and RN locations (above roof top) was performed iteratively with a radio planning and placement tool. The physical parameters and calculation steps are given in section III. In the end a coverage map was obtained which shows for each point on the map which serving station a terminal is best associated to (best server). This can be the base station, if this is the rate optimal association, but it can also be one of the relays $R N \in H 1$ or $R N \in H 2$. The maximum achievable rate at a certain point is then determined. From 


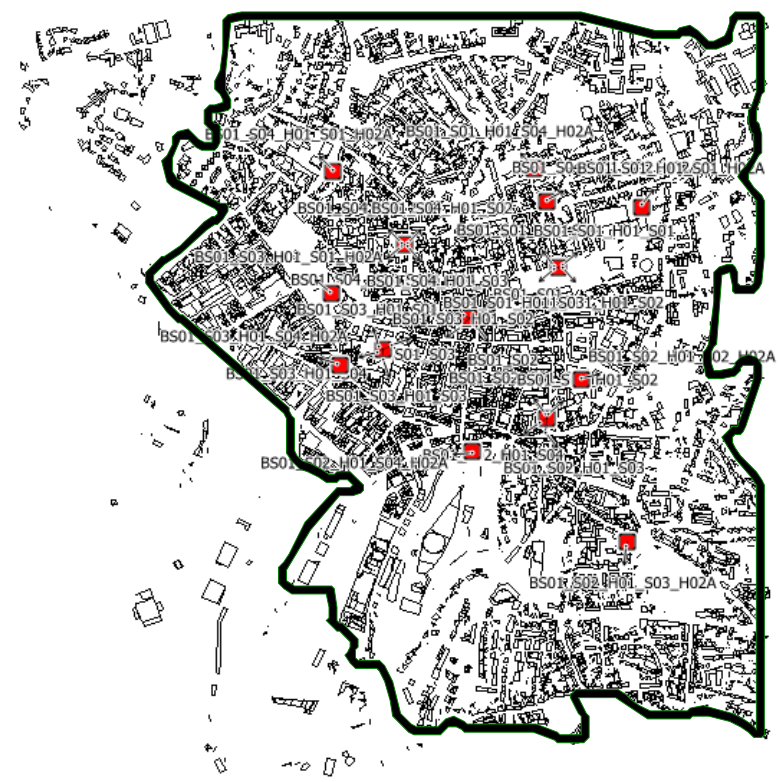

Fig. 1. The Scenario map of Jersey showing the BS (middle) and RN placement as well as the polygon of interest (green)

these matrix (2D) results we calculate scalar performance measures like the overall coverage and capacity, which are increased compared to using only one BS. Therefore we compare the scenario with 1) one BS only, 2) the BS plus a ring of four $R N \in H 1$ and 3) the BS plus a ring of four $R N \in H 1$ plus a second ring of the nine $R N_{2} \in H 2$.

\section{PHY AND DLC MODELS}

From link level to MAC throughput, the performance of the example system is evaluated by calculating the following steps.

- Transmit Power: $37 \mathrm{dBm}$ at the BS, $34 \mathrm{dBm}$ at the RN,

- Bandwidth: $b=18 \mathrm{MHz}$ net (20MHz system),

- Frequency: $2.5 \mathrm{GHz}$ apropriate for LTE or WiMAX,

- Pathloss I: 3D model of the city scenario (walls of buildings),

- Pathloss II: ray tracing to capture multi-path propagation,

- Noise: Thermal noise power is $N=-96.4 \mathrm{dBm}$,

- SINR: the first performance measure below PHY layer,

- MI: mutual information determined from SINR and modulation (Eq. 2),

- BER: bit error ratio, the PHY performance result,

- PER: packet error ratio, the result after channel decoding,

- Throughput: determined by bandwidth, PhyMode (modulation and code rate), ARQ overhead (Eq. 5),

- Second Hop Throughput: reduced by resources required on first hop (Eq. 6).

- Third Hop Throughput: reduced by resources required on first and second hop (Eq. 7).

\section{A. PHY layer}

The received power $P_{R, i}$ on every location is the output of software tools for raytracing. Indoor coverage was modeled by assuming wall thinkness and attenuation. The next

\begin{tabular}{|l|l|l|l|}
\hline PhyMode & modulation & code rate & $S I N R_{\min }$ in $[d B]$ \\
\hline 1 & QPSK & $1 / 3$ & 0.9 \\
2 & QPSK & $1 / 2$ & 2.1 \\
3 & QPSK & $2 / 3$ & 3.8 \\
4 & QAM16 & $1 / 2$ & 7.7 \\
5 & QAM16 & $2 / 3$ & 9.8 \\
6 & QAM16 & $5 / 6$ & 12.6 \\
7 & QAM64 & $2 / 3$ & 15.0 \\
8 & QAM64 & $5 / 6$ & 18.2 \\
\hline
\end{tabular}

TABLE I

LTE PhyModes AND THEIR REQUIRED $S I N R_{\text {min }}$

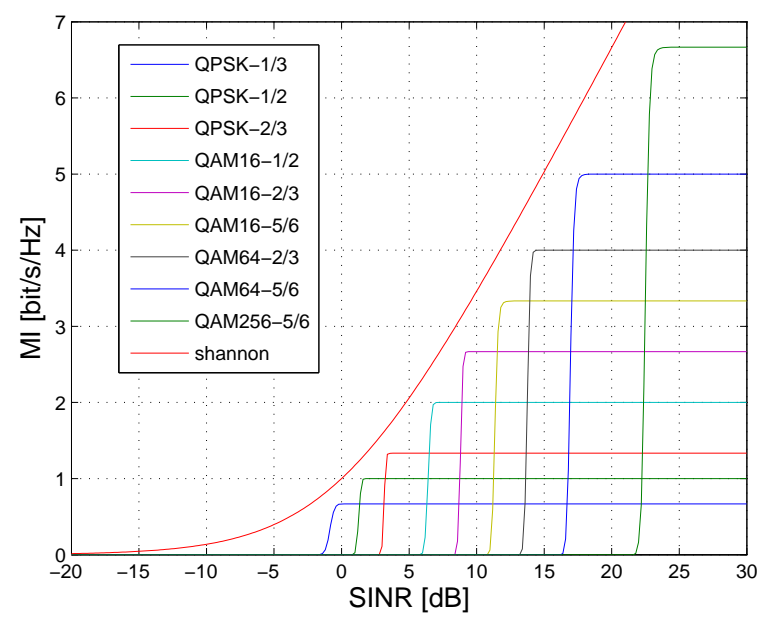

Fig. 2. Link level curves for the LTE modulation\&coding schemes (PhyMode). QAM256 is not used yet.

steps were analyzed analytically-numerically using Matlab. With $S I N R=P_{R, i} /(N+I)$ the signal to noise ratio is easily determined. For each $S I N R$ level between around 0 and 20 there in another PhyMode chosen, depending on the estimated performance of this PhyMode in terms of bis $/ s / H z$. For determining the required link level results we build upon the mutual information (MI) method [10]. We apply the steps $S I N R \rightarrow M I, M I \rightarrow B E R$ and $B E R \rightarrow P E R$ to get the packet error probability. For the $S I N R \rightarrow M I$ approximation. 2 was used [9]:

$$
\begin{gathered}
M I_{\text {shannon }}(S I N R)=\log _{2}\left(1+10^{S I N R / 10 d B}\right) \\
M I(S I N R, m)=\frac{1}{\left(\left[s \cdot M I_{\text {shannon }}(S I N R)\right]^{-w}+m^{-w}\right)^{1 / w}} \\
s=s(m)=0.95-0.08 \cdot(m \bmod 2) \\
w=w(m)=2 \cdot m+1
\end{gathered}
$$

$m$ is the modulation index, i.e. the number of bits per symbol ( $1=\mathrm{QPSK}, \ldots 8=\mathrm{QAM} 256)$. Figure 2 shows the outcome, taking also the coding rate into account to get the PHY throughput. LTE coders have rates $1 / 3,1 / 2,2 / 3$ and $5 / 6$ [11]. The PhyModes in this figure are given in Table I (QAM256 was not used). Within this cell, all $R N \mathrm{~s}$ are coordinated by the $B S$, so there is no intra-cell interference. 


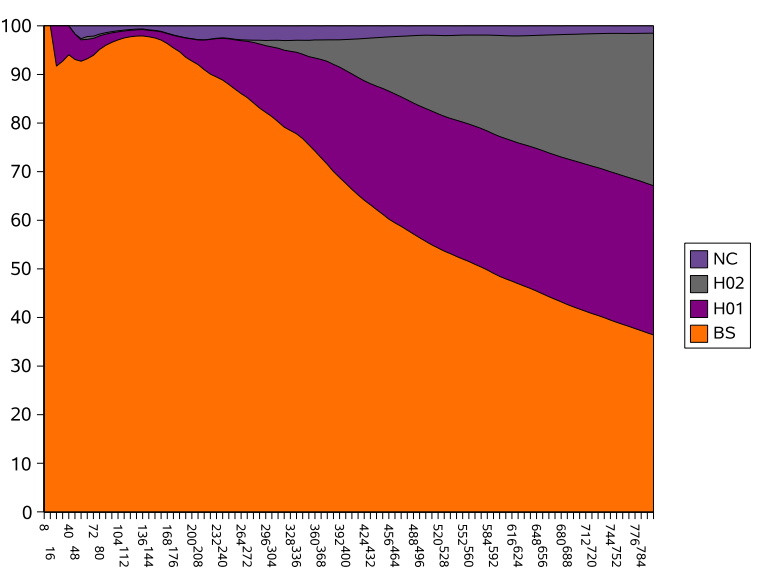

Fig. 4. The percentage of each station group used as a function of the cell radius from $0 . .800 m$

\section{B. DLC layer}

On DLC (MAC) layer, there is an overhead due to framing, signaling and ARQ retransmissions. The latter depends on $P E R$, which can be taken into account when assuming selective repeat ARQ by equation 5 .

$$
r_{\text {above } A R Q}=r_{\text {below } A R Q} \cdot(1-P E R)
$$

In total we obtain a MAC overhead of $M A C / P H Y=$ $132.3 \%$. The PHY overhead of PHY/RAW $=107.1 \%$ comes from OFDM cyclic prefix duration. Under multihop operation there are individual resources needed on every hop. The constant packet length requires a different resource share depending on the used PhyMode which determines the maximum rate $r_{i, \max }$ usable on each hop. Therefore we can get the maximum rate on the second hop to be

$$
r_{2}=\left(r_{1, \max }^{-1}+r_{2, \max }^{-1}\right)^{-1}
$$

and on the third hop

$$
r_{3}=\left(r_{1, \text { max }}^{-1}+r_{2, \text { max }}^{-1}+r_{3, \text { max }}^{-1}\right)^{-1}
$$

For every location $(x, y)$ we can now determine the best rate out of $r_{1}, r_{2}, r_{3}$ which gives us the result in Figure 7 . One of the three rates is maximum and the index $i$ of the maximum $r_{i}$ determines the "best server", i.e. it shows which station the UT at that location should be associated with.

The performance results have been obtained over different areas. First, the full area was used, including less densely populated parts ("square"). Second, a circular area with a radius of $800 \mathrm{~m}$ around the BS was defined, which gives a realistic cell size ("circle"). Third, the area within a polygon (Fig. 1) was studied (the urban populated area).

\section{AnAlysis RESUlts}

The analysis has been carried out to generate the twodimensional data in fig. 6 and 7. Scalar results were only counted in the polygon area defined in Fig. 1. Here we show the downlink only that also applies to the uplink if the uplink pathloss is the same (FDD). But the benefit in terms of capacity reveals if we derive scalar performance measures

\begin{tabular}{|l|l|l|l|}
\hline Scenario & coverage [\%] & capacity $[$ Mbit/s] & spec.eff. $[$ bit $/$ s $/ \mathrm{Hz}]$ \\
\hline BS only & 47.257 & 16.223 & 0.901 \\
BS+H1 & 79.891 & 18.217 & 1.012 \\
BS+H1+H2 & 98.519 & 21.583 & 1.199 \\
\hline
\end{tabular}

TABLE II

SCALAR RESULTS FOR THE RELAY SCENARIO WITHIN THE SQUARE AREA

\begin{tabular}{|l|l|l|l|}
\hline Scenario & coverage $[\%]$ & capacity $[$ Mbit/s] & spec.eff. [bit/s/Hz] \\
\hline BS only & 64.816 & 18.519 & 1.029 \\
BS+H1 & 87.536 & 22.091 & 1.227 \\
BS+H1+H2 & 98.925 & 24.375 & 1.354 \\
\hline
\end{tabular}

TABLE III

SCALAR RESULTS FOR THE RELAY SCENARIO WITHIN THE POLYGON AREA

from it. The coverage (in $\%$ of the area) of the scenarios differing by the number of relays involved is determined by counting all locations with $S I N R>S I N R_{\min }$. For LTE $S I N R_{\text {min }}=0.9 \mathrm{~dB}$ holds. Figure 3(a) shows the coverage of each scenario. The system capacity is determined by assuming equal traffic load for each user terminal and a homogeneous user density over the area. This means that a UT far outside, having a low PhyMode, requires more share of the resources than a UT close to the BS. The following equation [12] for the capacity $C$ considers this:

$$
\frac{1}{C}=\int_{\text {polygonarea }} \frac{1}{\operatorname{Capacity}(x, y)} d x d y
$$

Figure 3(b) shows the capacity of each scenario. Figure 5 shows that in a multihop scenario, more and more of the coverage area of $B S$ is taken over by $R N s$. The capacity $C$ in $\mathrm{bit} / \mathrm{s}$ can be used to calculate the spectral efficiency $e=C / b$ using the used bandwidth $b$. The performance metrics are shown in Table II for the full area and in Table III for the densely populated polygon. According to this, two tiers of relays, compared to the BS only scenario, increase the coverage by a factor of 2.08 overall and 1.53 within the city, and both the capacity and spectral efficiency by a factor of approximately 1.33 both overall and within the city. Figure 4 shows the partition of area which is served by either BS, H1 or $\mathrm{H} 2$ dependent on the radius of the cell. We observe, that a first ring of relays comes in early, and a second ring is worth to use beginning from $350 \mathrm{~m}$. These numbers show the benefit of using relays. The radio power sum is of course more here, but the expensive fiber access to the BS is saved, which is the limitation in early rollout phases.

\section{CONCLUSION}

In this paper a realistic urban scenario was treated offering numeric results for the coverage and capacity of a multihop radio cell. We analyzed the topologic structure of the city to get the path loss, SINR and data rate values for every location in that area. Next the coverage (in \%) and capacity (in $\mathrm{Mbit} / \mathrm{s}$ ) as well as the spectral efficiency were derived from that, calculated for the whole system, but counted only within a bounded polygon area. Doing this analysis for three scenarios, one with a base station only, one with two hops (one tier of relays) and one with three hops (two tiers of relays), 


\section{Coverage}

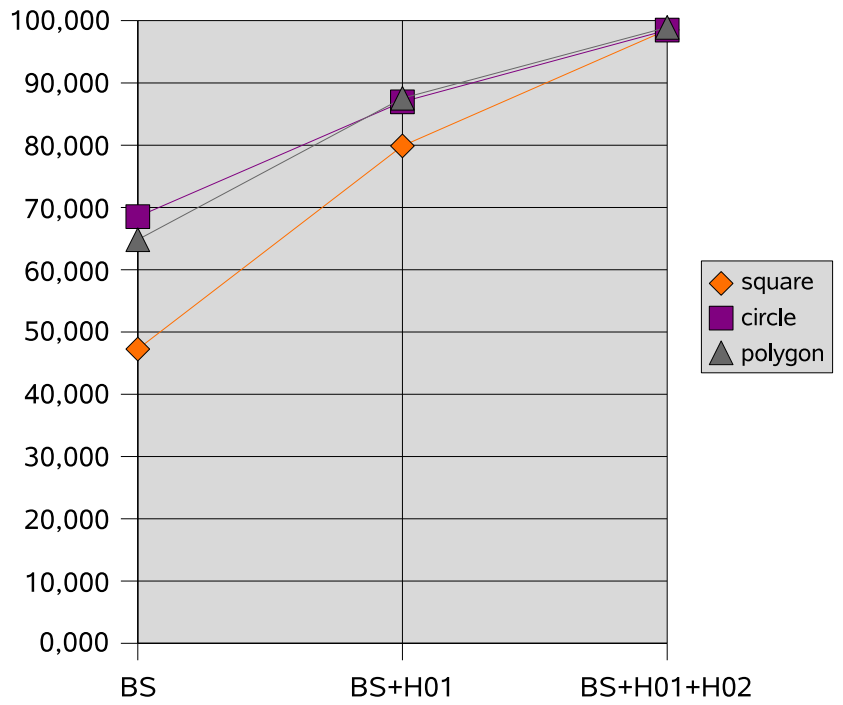

(a) coverage using relays

\section{Capacity [Mbit/s]}

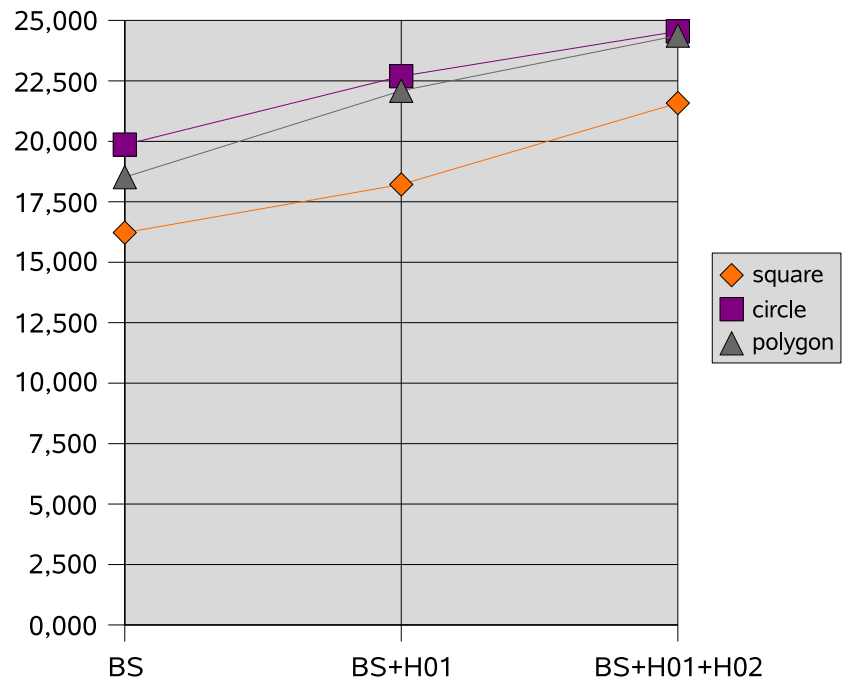

(b) capacity using relays

Fig. 3. Coverage and capacity compared for three scenarios: BS (one BS only), BS+H01 (with one tier of relays), BS+H01+H02 (BS with two relay hops $\mathrm{H} 1+\mathrm{H} 2)$. Three different integration areas are the parameter.

\section{coverage for best server}

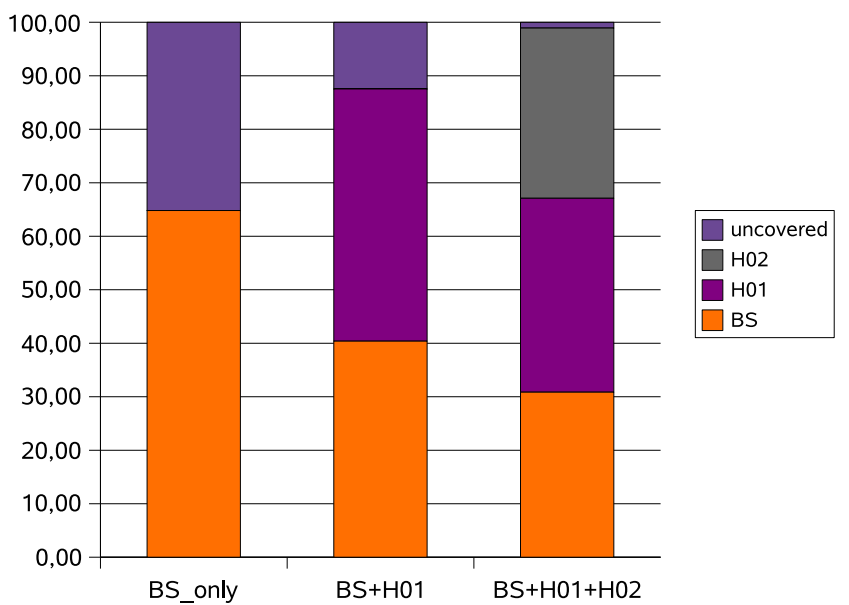

Fig. 5. The fraction of area served by each group of servers, determined within the polygon area

remarkable gains were visible when using relays. There is only one interface to the fixed network in all three scenarios. All the relays help to cover areas behind obstructions (buildings) where the path loss from the BS would be too large. The multihop techniques also improve the spectrum efficiency, because for all covered areas there is only one radio channel needed. The alternative was to place several BSs to serve the same area. But this requires more channels, since a cluster order of one suffers from too much interference. One outlook is an economic comparison of the CAPEX and OPEX related to the scenarions, e.g. [13]. Another outlook is the analysis of multiple cells with interference effects at the border.

\section{REFERENCES}

[1] http://www.3gpp.org/Highlights/LTE/LTE.htm.

[2] http://www.wimaxforum.org/.

[3] R. Pabst, B. Walke, D. C. Schultz, and et al, "Relay-Based Deployment Concepts for Wireless and Mobile Broadband Radio," IEEE Communications Magazine, pp. 80-89, Sep 2004.

[4] V. Sreng, H. Yanikomeroglu, and D. Falconer, "Coverage enhancement through two-hop relaying in cellular radio systems," in Proc. IEEE Wireless Communications and Networking Conference(WCNC'02), 2002.

[5] H. Yanikomeroglu, "Fixed and mobile relaying technologies for cellular networks," in 2nd Workshop on Applications and Services in Wireless Networks (ASWN'02), 3-5 July 2002, pp. 75-81.

[6] R. Schoenen, J. Eichinger, and B. Walke, "On the OFDMA FDD mode in 3G-LTE," in Proceedings of the 12th International OFDM Workshop (InOWo'07), Hamburg, Germany, Aug 2007. [Online]. Available: http://www.comnets.rwth-aachen.de

[7] R. Schoenen, W. Zirwas, and B. Walke, "Capacity and Coverage Analysis of a 3GPP-LTE Deployment Scenario," in Proceedings of the Broadband Wireless Access Workshop BWAW'08 colocated with ICC'08, Beijing, China, May 2008. [Online]. Available: http://www.comnets.rwth-aachen.de

[8] M. Lott, M. Weckerle, and et al, "Hierarchical cellular multihop networks," in Proceedings of the IEEE EPMCC'03, 2003.

[9] R. Schoenen and B. Walke, "On PHY and MAC performance of 3G-LTE in a multi-hop cellular environment," in Proceedings of the 3rd IEEE International Conference on Wireless Communications, Networking and Mobile Computing (WiCOM), Shanghai, China, Sep 2007. [Online]. Available: http://www.comnets.rwth-aachen.de

[10] K. Brueninghaus and D. e. a. Astely, "Link performance models for system level simulations of broadband radio access systems," in Proceedings of the 17th Annual IEEE International Symposium on Personal, Indoor and Mobile Radio Communications, Sep 2005, pp. 2306-2311.

[11] K. J. e. a. Ekstrom H., Furuskar A., "Technical solutions for the 3G long-term evolution," IEEE Communications Magazine, pp. 38-45, Mar 2006.

[12] C. Hoymann and S. Goebbels, "Dimensioning cellular wimax part i: Singlehop networks," in Proceedings of European Wireless 2007, Paris, France, Apr 2007, p. 7. [Online]. Available: http: //www.comnets.rwth-aachen.de

[13] Bogdan Timus, "Deployment Cost Efficiency in Broadband Delivery with Fixed Wireless Relays,” Ph.D. dissertation, KTH Stockholm, 2006. 


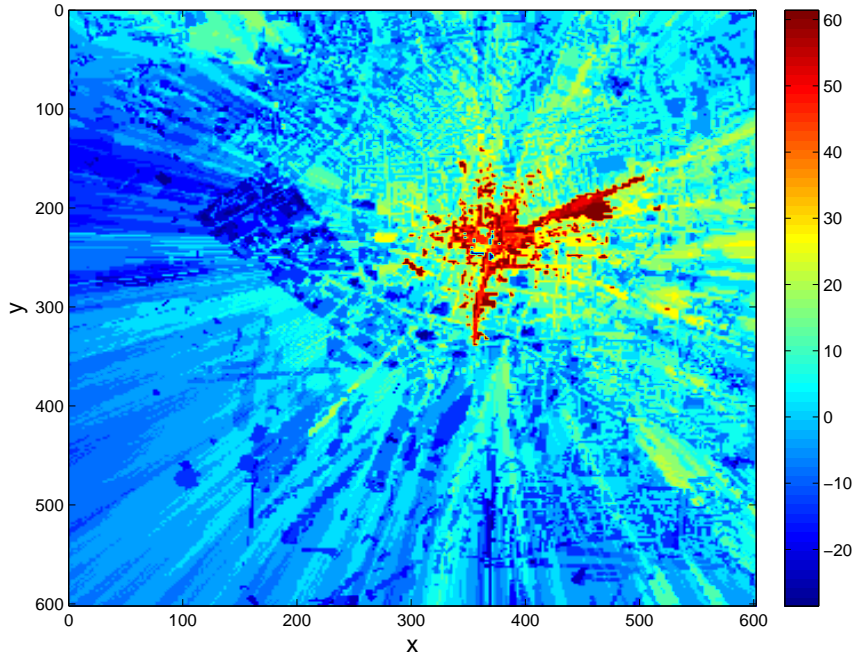

(a) SINR of BS only (in $d B$ )

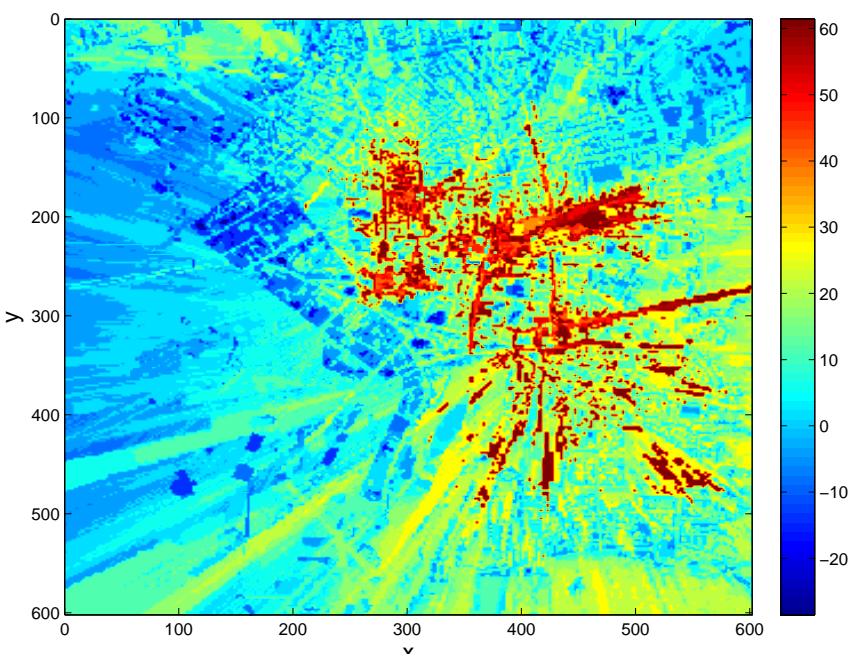

(c) SINR of BS with H1 (in $d B$ )

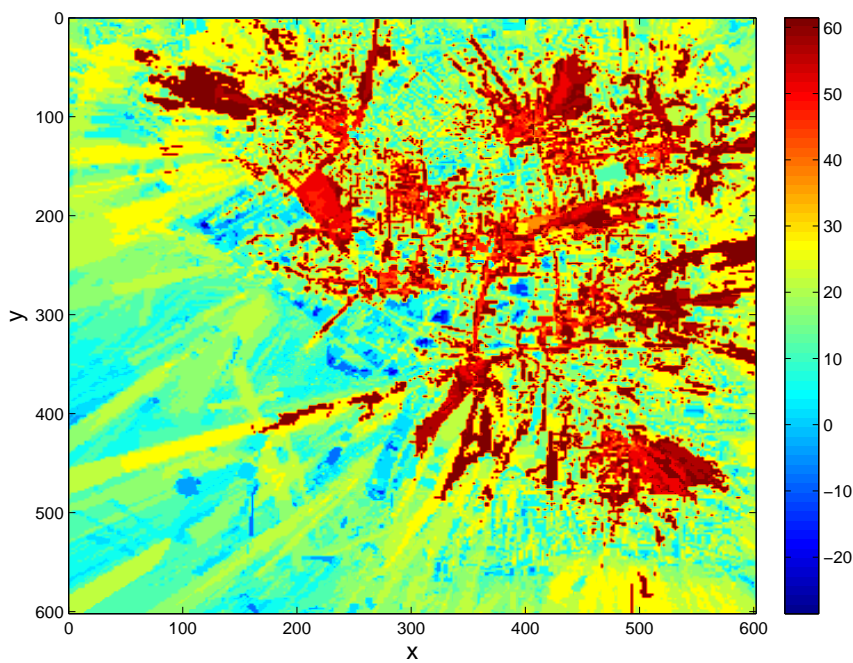

(e) SINR of BS with $\mathrm{H} 1$ and $\mathrm{H} 2$ (in $d B$ )

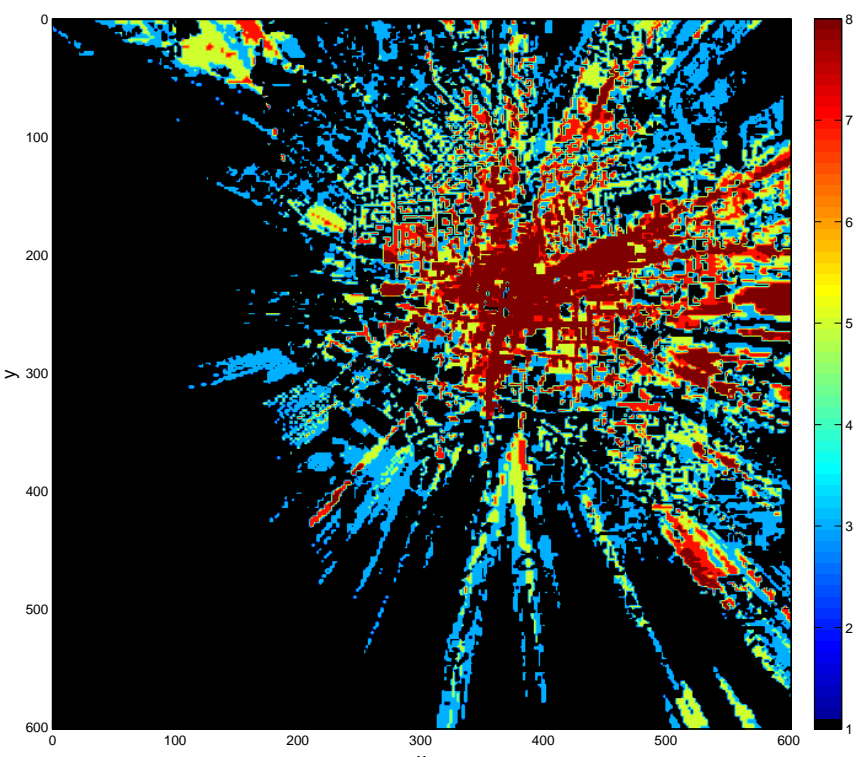

(b) PhyMode of BS only

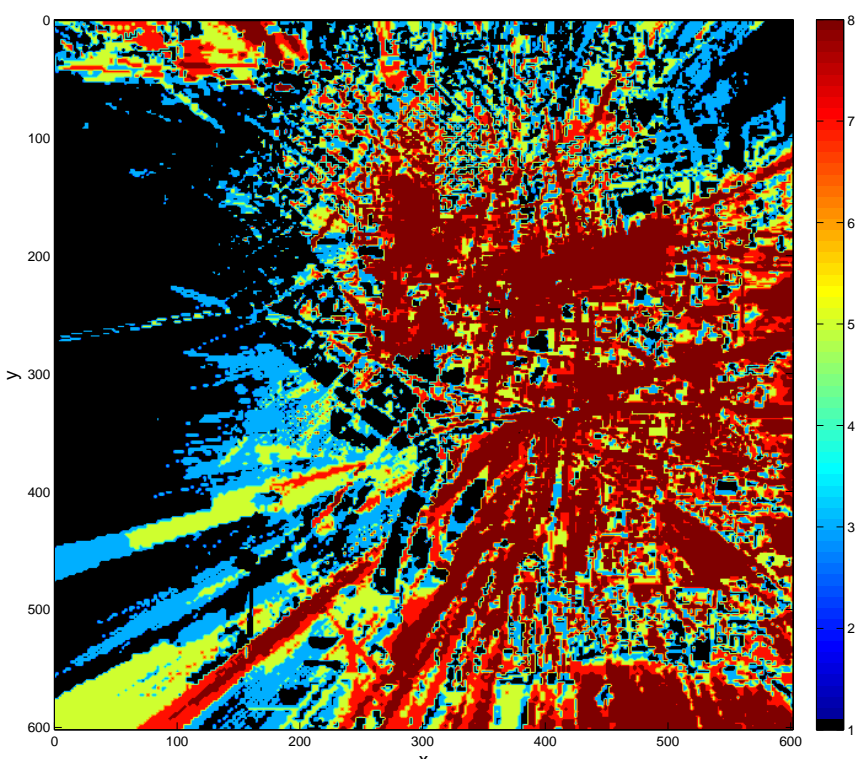

(d) PhyMode of BS with H1

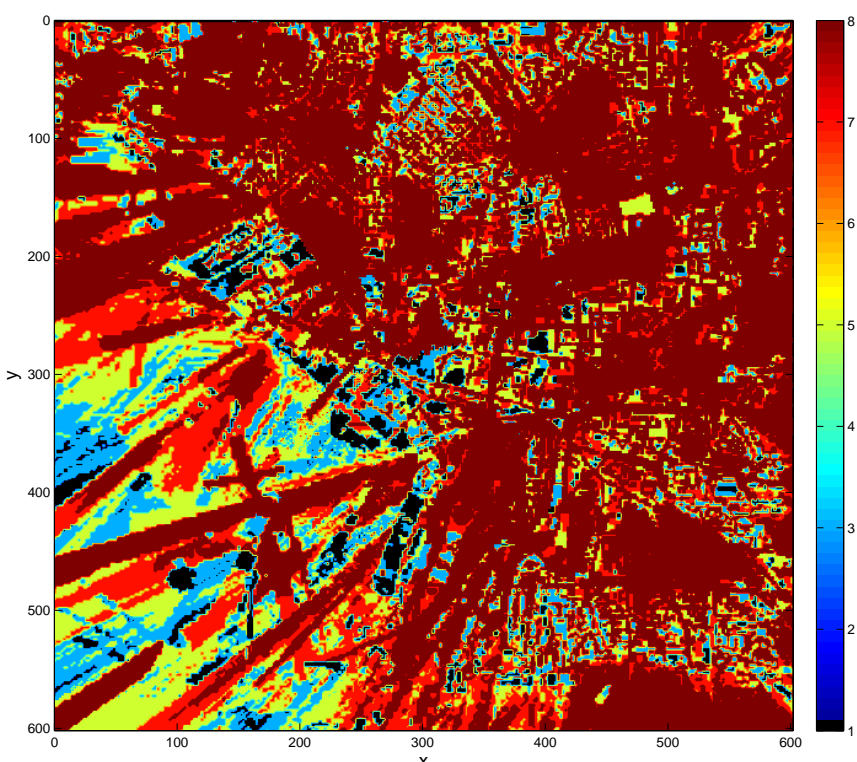

(f) PhyMode of BS with H1 and H2

Fig. 6. On the area map of Jersey, these figures show the SINR [dB] and PhyMode [1..8] for a scenario with BS only, with one tier of relays H1, and with two relay hops $\mathrm{H} 1+\mathrm{H} 2$ 


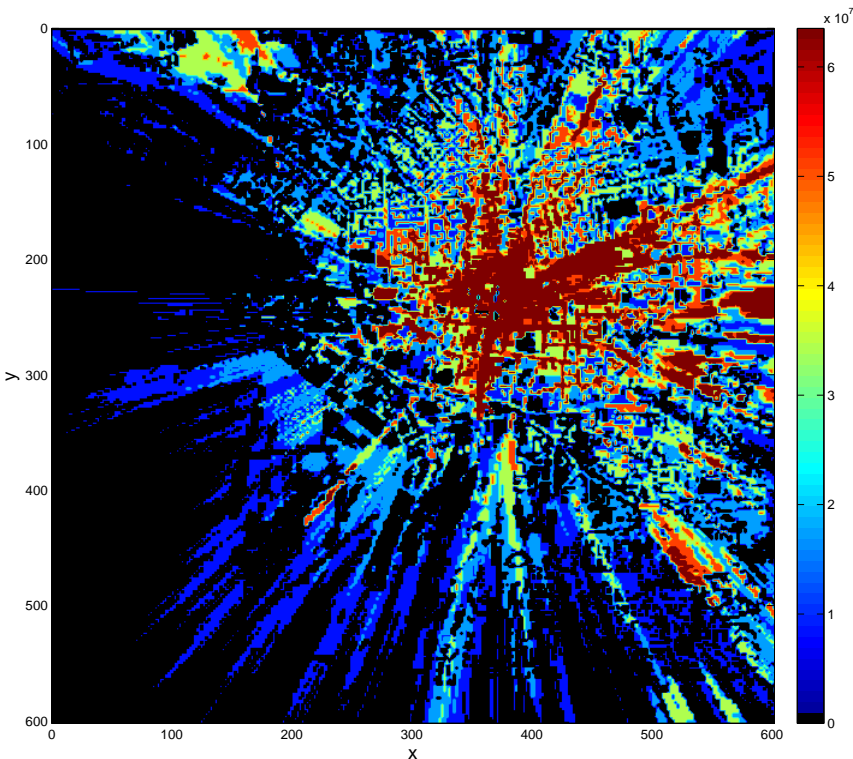

(a) Rate of BS only

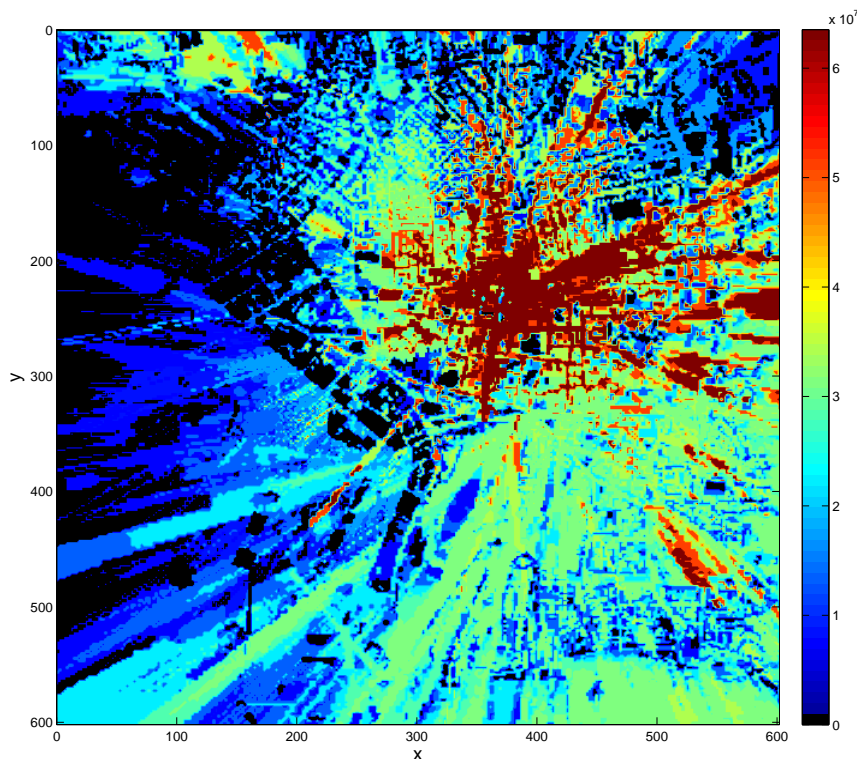

(c) Rate of BS with $\mathrm{H} 1$

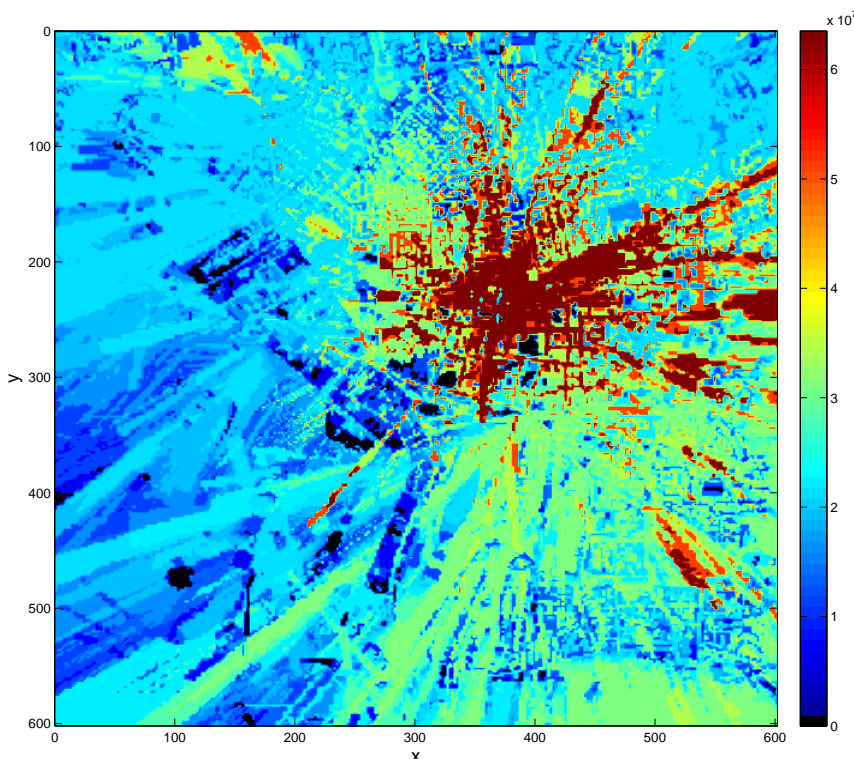

(e) Rate of BS with $\mathrm{H} 1$ and $\mathrm{H} 2$

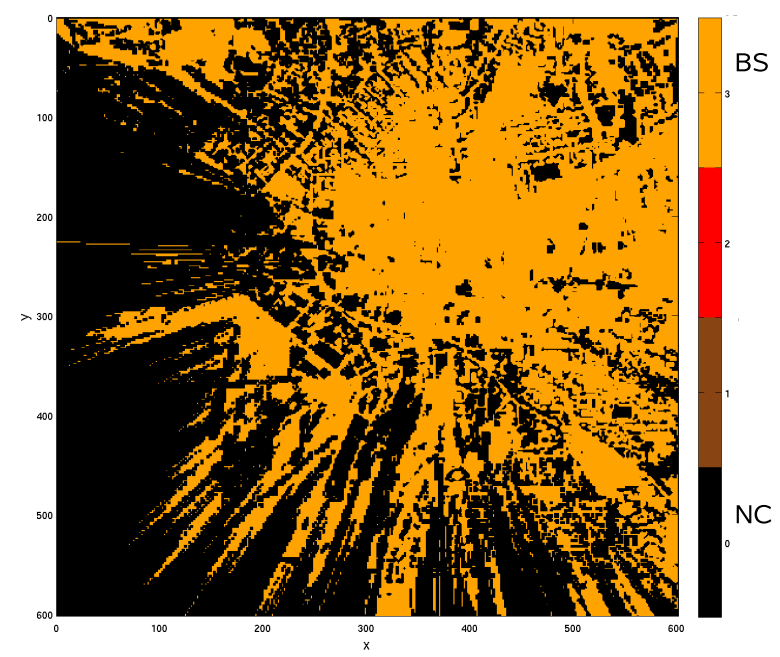

(b) Best server (coverage) of BS only

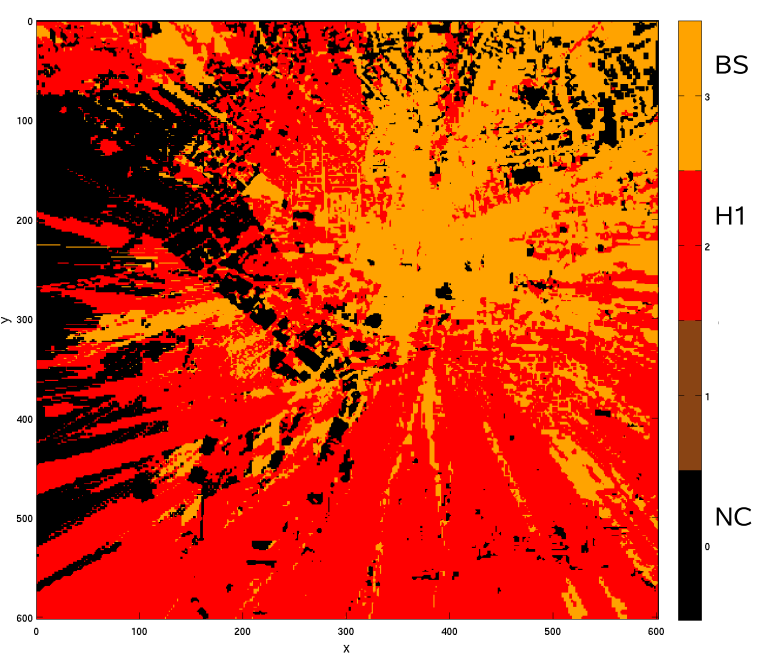

(d) Best server (coverage) of BS with H1

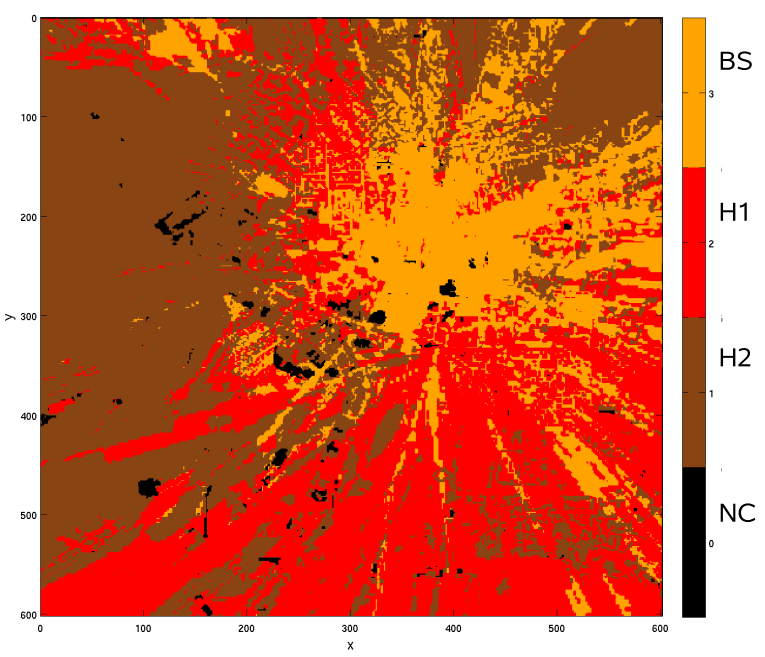

(f) Best server (coverage) BS with $\mathrm{H} 1$ and $\mathrm{H} 2$

Fig. 7. On the area map of Jersey, these figures show the available rate capacity $[\mathrm{bit} / \mathrm{s}]$ and best server $($ middle=3=BS, $2=\mathrm{H} 1,1=\mathrm{H} 2)$ for a scenario with BS only, with one tier of relays $\mathrm{H} 1$, and with two relay hops $\mathrm{H} 1+\mathrm{H} 2$ 